NRTSC

Technology

On Time

On Target

NUCLEAR REACTOR TECHNOLOGY AND SCIENTIFIC COMPUTATIONS
W S R C - T R - 91 - 481

KEY WORDS: Diffusion Theory

Few Group Infinite Lattice

RETENTION PERIOD: PERMANENT

\title{
USER'S MANUAL FOR GILDA: \\ An Infinite Lattice \\ Diffusion Theory Calculation (U)
}

B y

T. T. Le

ISSUED: NOVEMBER 1991 
PROJECT: PHYSICS CODE CERTIFICATION

TASK: CERTIFICATION OF GILDA

DOCUMENT: WSRC-TR-91.481

TITLE: USER'S MANUAL FOR GILDA: An Infinite Lattice Diffusion Theory Calculation (U)

\section{APPROVALS}

$\frac{\text { h } \& \text { - zrost }}{\text { R.L. FROST, TECHNICAL REVIEWER }}$

DATE: $11 / 19 / 91$

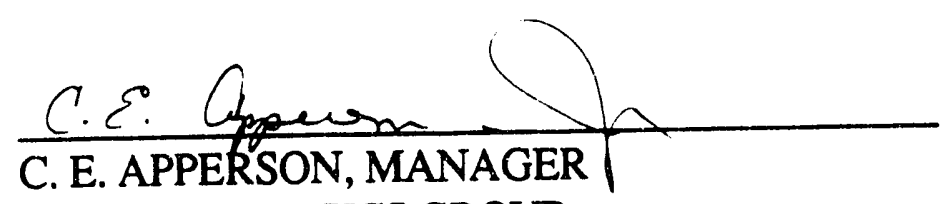

DATE: $12 \cdot 4-91$ REACTOR PHYSICS GROUP

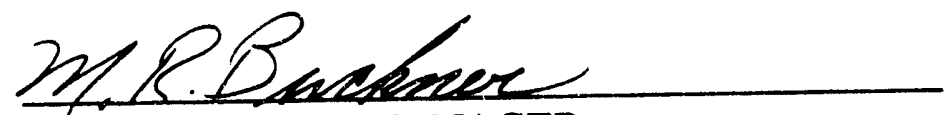

DATE: $12-10-91$

M.R. BUCKNER, MANAGER

SCIENTIFIC COMPUTATIONS SECTION 


\section{TABLE OF CONTENTS}

P a g e

Table of Contents

I. Introduction

03

II. Theory

03

II.1 Static Multigroup Neutron Diffusion Equation

03

II.2 Finite Difference Approximation

05

III. Method of Solution

III. 1 Outer Iteration

III. 2 Inner Iteration

IV. Program's Subroutines

V. Input Description

VI. Output Description

VI.1 JOSHUA Output Records

VI.2 Additional Outputs

Appendix A: Table and Figures

A. 1 Table

A.2 Figures

Appendix B: Running GILDA on VAX/VMS and IBM Systems 30 
B.1 Running GILDA on VAX/VMS

B.2 Running GILDA on IBM $\ldots \ldots$

Appendix C: Sample Input Records 33

References 
User's Manual for GuLd (U)

\section{INTRODUCTION}

GILDA is a static two-dimensional diffusion theory code that performs either buckling $\left(B^{2}\right)$ or k-effective $\left(k_{e f f}\right)$ calculations for an infinite hexagonal lattice which is constructed by repeating identical seven-cell zones (one cell is one or seven identical homogenized hexes). GILDA was written by J. W. Stewart ${ }^{1}$ in 1973.

This user's manual is intended to provide all of the information necessary to set up and execute a GILDA calculation and to interpret the output results. It is assumed that the user is familiar with the computer (VAX/VMS or IBM/MVS) and the JOSHUA system database on which the code is implemented. Users who are not familiar with the JOSHUA database are advised to consult Reference 2 to understand the structure of JOSHUA records and data sets before turning to section 4 of this manual.

Sections 2 and 3 of this manual serve as a theory document in which the basic diffusion theory and the numerical approximations behind the code are described. Section 4 describes the functions of the program's subroutines. Section 5 describes the input data and tutors the user how to set up a problem. Section 6 describes the output results and the error messages which may be encountered during execution. Users who only wish to learn how to run the code without understanding the theory can start from section 4 and use sections 2 and 3 as references. Finally, the VAX/VMS and the IBM execution command files together with sample input records are provided in the appendices at the end of this manual.

\section{THEORY}

\section{II.1 Static Multigroup Neutron Diffusion Equations}

The basic static, continuous energy-space, two dimensional neutron diffusion equation ${ }^{3}$ is: 
User's Manual for GULDA (U)

$$
\begin{array}{r}
-\nabla D(\vec{r}, E) \cdot \nabla \phi(\vec{r}, E)+\left[\Sigma_{a}(\vec{r}, E)+\Sigma_{s}(\vec{r}, E)+D(\vec{r}, E) B^{2}\right] \phi(\vec{r}, E)= \\
\int_{E^{\prime}}\left\{\frac{\chi\left(\vec{r}, E^{\prime} \rightarrow E\right)}{k_{e f f}} \nu \Sigma_{f}\left(\vec{r}, E^{\prime}\right)+\Sigma_{s}\left(\vec{r}, E^{\prime} \rightarrow E\right)\right\} \phi\left(\vec{r}, E^{\prime}\right) d E^{\prime}
\end{array}
$$

where the parameters are defined as follows:

D Diffusion coefficient

$\vec{r} \quad$ Position vector

E Neutron energy

$\phi \quad$ Neutron flux

$\Sigma_{\mathrm{x}} \quad$ Neutron macroscopic cross sections with $\mathrm{x}=\mathrm{a}$ for absorption, $f$ for fission, $c$ for capture, $r$ for removal, and $s$ for scattering

$\chi \quad$ Fission neutron spectrum

$\checkmark \quad$ Average number of neutrons released per fission event

$k_{e f f}$ Effective multiplication factor

$\mathrm{B}^{2} \quad$ Buckling

To simplify the energy space diffusion equations, the energy range of neutrons is divided into $G$ groups of energy; in each group, the diffusion coefficient, cross sections, and emission spectrum are assumed to be constants. The highest energy level is $E_{0}$ and the lowest is $E_{G}$; group $g$ has energies between $E_{g}$ and $E_{g-1}$. With the assumption of constant group parameters in each group, the group parameters are the average values in each group, and the multigroup neutron diffusion equation for group $\mathrm{g}$ is:

$$
\begin{gathered}
-\nabla D^{g}(\vec{r}) \cdot \nabla \phi^{g}(\vec{r})+\left[\Sigma_{a}^{g}(\vec{r})+\Sigma_{s}^{g}(\vec{r})+D^{g}(\vec{r}) B^{2}\right] \phi^{g}(\vec{r})= \\
\left.\sum_{g^{\prime}}^{G}\left\{\frac{\chi^{g} \rightarrow g(\vec{r})}{k_{e f f}} v \Sigma_{f}^{g}(\vec{r})+\Sigma_{s}^{g} \rightarrow g_{(\vec{r})}\right)\right\} \phi^{g}(\vec{r})
\end{gathered}
$$


where

$$
\begin{aligned}
& \phi^{g}(\vec{r})=\int_{E_{g}}^{E_{g-1}} \phi(\vec{r}, E) d E \\
& D^{g}(\vec{r}) \nabla \overrightarrow{g^{g}(\vec{r})}=\int_{E_{g}}^{E_{g-1}} D(\vec{r}, E) \nabla \phi(\vec{r}, E) d E \\
& \Sigma_{x}^{g}(\vec{r})=\frac{\int_{E_{g}}^{E_{g-1}} \Sigma_{x}(\vec{r}, E) \phi(\vec{r}, E) d E}{\phi^{g}(\vec{r})} \\
& \Sigma_{x}^{g} \rightarrow g(\vec{r})=\frac{\int_{E_{g}}^{E_{g-1}} \int_{E_{g^{\prime}}}^{E_{g^{\prime}-1}} \Sigma_{x}\left(\vec{r}, E^{\prime} \rightarrow E\right) \phi(\vec{r}, E) d E d E^{\prime}}{\chi^{g}(\vec{r})} \\
& \dot{\chi^{g} \rightarrow g(\vec{r})}=\int_{E_{g}}^{E_{g-1}} \int_{E_{g}^{\prime}}^{E_{g^{\prime}-1}} \chi\left(\vec{r}, E^{\prime} \rightarrow E\right) d E d E^{\prime}
\end{aligned}
$$

Equation (2.2) can be simplified to:

$$
-\nabla D^{g}(\vec{r}) \cdot \nabla \phi^{g}(\vec{r})+R^{g}(\vec{r}) \phi^{g}(\vec{r})=P^{g}(\vec{r})
$$

where $R^{g}$ is defined as the removal cross section and $P g$ is the production rate for neutron group $\mathrm{g}$ :

$$
\begin{aligned}
& R^{g}(\vec{r})=\Sigma_{a}^{g}(\vec{r})+\left(\sum_{g \neq g}^{G} \Sigma_{s}^{g \rightarrow g^{\prime}}(\vec{r})\right)+D^{g}(\vec{r}) B^{2}=\Sigma_{r}^{g}(\vec{r})+D^{g}(\vec{r}) B^{2} \\
& P^{g}(\vec{r})=\sum_{g=1}^{G} \frac{\chi^{g} \rightarrow g(\vec{r})}{k_{e f f}} \vee \Sigma_{f}^{g}(\vec{r}) \phi^{g^{\prime}(\vec{r})}+\sum_{g=g}^{G} \Sigma_{s}^{g \rightarrow g}(\vec{r}) \phi^{g}(\vec{r})
\end{aligned}
$$




\section{II.2 Finite Difference Approximation}

To solve the multigroup-space neutron diffusion equation, the continuous spatial distribution is discretized into finite volume elements called meshes. Associated with each mesh is a mesh point centered within the mesh and the material parameters in each mesh are homogenized. For a mesh $\mathrm{i}$, integrating equation (2.3) over its volume we have:

$$
-\frac{1}{V_{i}} \int_{V_{i}} \nabla D^{g}(\vec{r}) \cdot \nabla \phi^{g}(\vec{r}) d r+R_{1}^{g} \phi_{i}^{-g}=P_{1}^{g}
$$

or

$$
\frac{1}{V_{i}} \int_{V_{i}} \nabla \cdot J g(\vec{r}) d r+R_{1}^{g} \stackrel{-g}{\phi}_{i}=P_{1}^{g}
$$

where $\mathrm{J}^{\mathrm{g}}$, according to Fick's law, is the total neutron current of group g:

$$
\begin{aligned}
& J^{g}(\vec{r})=-D^{g}(\vec{r}) \nabla \phi^{g}(\vec{r}) ; \quad \stackrel{-g}{\phi_{i}}=\frac{1}{V_{i}} \int_{V_{i}} \phi^{g}(\vec{r}) d \vec{r} ; \\
& P_{1}^{g}=\frac{1}{V_{i}} \int_{V_{i}} P^{g}(\vec{r}) d \vec{r} ; \quad R_{1}^{g} \stackrel{-g}{\phi_{i}}=\frac{1}{V_{i}} \int_{V_{i}} R^{g}(\vec{r}) \phi^{g}(\vec{r}) d \vec{r}
\end{aligned}
$$

Applying Gauss's law to the leakage term in equation (2.5) with $S_{i}$ as the total surface area of mesh $i$, results in:

$$
\frac{1}{V_{i}} \int_{V_{i}} \nabla \cdot J^{g}(\vec{r}) d \vec{r}=\frac{1}{V_{i}} \int_{S_{i}}\left[J^{g}(\vec{r}) \cdot \vec{n}\right] d S
$$

Consider a mesh $\mathrm{i}$ surrounded by $\mathrm{N}$ neighboring meshes, $\mathrm{n}$, as shown in Figures 2 through 7 in the Appendix A. The boundary surfaces between mesh $i$ and $n$ are defined as $A_{i n}$, and the distances from the center of mesh $i$ and $n$ to the surface $A_{\text {in }}$ are $1 / 2 h_{i}$ and $1 / 2 h_{n}$ respectively. If the surfaces are flat (the surface lies entirely in a plane), the integral in equation (2.6) can be substituted by a 
summation over all boundary surfaces of the mesh, such that:

$$
\frac{1}{V_{i}} \int_{S_{i}}\left[J^{g}(r) \cdot n\right] d S=\frac{1}{V_{i}} \sum_{n=1}^{N} J_{i n}^{g} A_{i n}=\sum_{n=1}^{N} \frac{A_{i n}}{V_{i}} J_{i n}^{g}
$$

Approximating the neutron current by the standard finite difference, we have:

$$
\left.J_{i n}^{g}\right|_{i}=-D_{1}^{g} \frac{2\left(\phi_{i n}^{g}-\phi_{i}^{g}\right)}{h_{i}} ;\left.\quad J_{i n}^{g}\right|_{n}=-D_{n}^{g} \frac{2\left(\phi_{n}^{g}-\phi_{i n}^{g}\right)}{h_{n}}
$$

where $\phi_{1}^{g}, \phi_{n}^{g}$, and $\phi_{1 n}^{g}$ are the neutron point flux values at the center of mesh $i$, center of mesh $n$, and boundary interface $A_{i n}$ respectively.

The continuity condition at the mesh interface $A_{\text {in }}$ gives $\left.\mathrm{J}_{\mathrm{in}}^{\mathrm{g}}\right|_{\mathrm{i}}=\left.\mathrm{J}_{\mathrm{in}}^{\mathrm{g}}\right|_{\mathrm{n}}=\mathrm{J}_{\mathrm{in}}^{\mathrm{g}}$, that is:

$$
\begin{aligned}
& J_{i n}^{g}=\left.J_{i n}^{g}\right|_{i}=-D_{i}^{g} \frac{2\left(\phi_{i n}^{g}-\phi_{i}^{g}\right)}{h_{i}} \\
& J_{i n}^{g}=\left.J_{i n}^{g}\right|_{n}=-D_{n}^{g} \frac{2\left(\phi_{n}^{g}-\phi_{i n}^{g}\right)}{h_{n}}
\end{aligned}
$$

where $\left.J_{\text {in }}^{g}\right|_{n},\left.J_{i n}^{g}\right|_{i}$ refer to the currents to mesh $i$ from the interface $A_{\text {in }}$ which are evaluated by the interface flux $\phi_{\text {in }}$ with the point flux in mesh $\mathrm{n}, \mathrm{i}$ respectively. Solve one of equations (2.9) for the interface flux $\phi_{i n}$ in terms of center point fluxes $\phi_{i}, \phi_{n}$ and substitute this back into another equation (2.9); the neutron current at the interface $A_{\text {in }}$ then becomes:

$$
J_{\text {In }}^{g}=\frac{2}{\frac{h_{i}}{D_{1}^{g}}+\frac{h_{n}}{D_{n}^{g}}}\left(\phi_{1}^{g}-\phi_{n}^{g}\right)
$$


For a regular lattice with equal meshes, $h_{i}=h_{n}=h$; substituting equations (2.10) and (2.7) for leakage term into equation (2.5) results in a standard finite difference equation for neutron group $g$, mesh i:

$$
\sum_{n=1}^{N} C_{i n}^{g}\left(\phi_{1}^{g}-\phi_{n}^{g}\right)+R_{i}^{g} \stackrel{-g}{\phi_{i}}=P_{i}^{g}
$$

where

$$
\mathrm{C}_{\mathrm{in}}^{\mathrm{g}}=\frac{\mathrm{A}_{\mathrm{in}}}{\mathrm{hV}_{\mathrm{i}}} \frac{2}{\left(\frac{1}{D_{\mathrm{g}}^{\mathrm{g}}}+\frac{1}{D_{\mathrm{n}}^{\mathrm{g}}}\right)}
$$

In GILDA, the standard finite difference equation (2.11) is used with the assumption that mesh average fluxes equal mesh centered point flux values. The program was coded for a zone of sevenhomogenized cells in which each cell is a hex or seven identical hexes with either one, three, or six mesh points (meshes) per hex. The infinite lattice is defined by repeating these zones with either translational symmetry or translation and rotational symmetry.

Since the approximation of centered point flux values for mesh average fluxes and the first order finite difference from center to the edge of the mesh is not accurate for highly heterogeneous lattices in which the flux may vary stiffly, another finite difference method called the Coarse Mesh (CM) method $^{4}$ was also implemented. This method assumes that the shape of the fluxes is linear from the boundary interface to a distance $\mu \mathrm{h} / 2$ toward the center of the mesh and flat in the remaining central sub-mesh as shown in figure 8 in Appendix A, that is: 
User's Menual for GULDA (U)

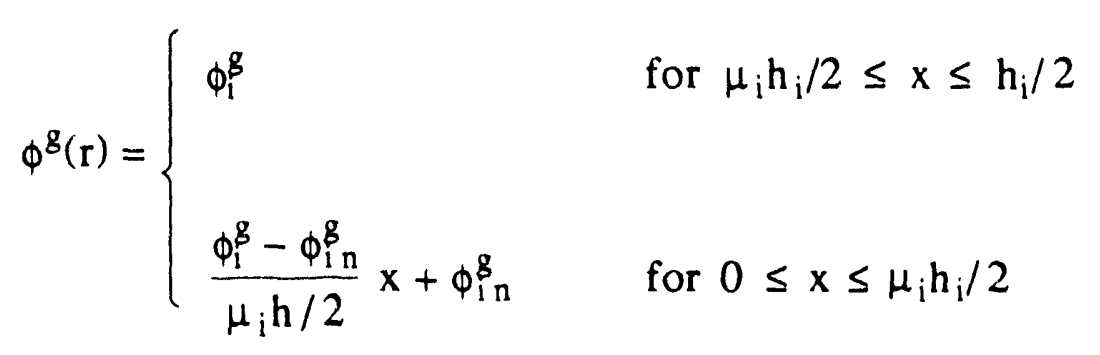

Applying the same procedure as above with $h_{i}$ and $h_{n}$ replaced by $\mu_{\mathrm{i}} \mathrm{h}_{\mathrm{i}}$ and $\mu_{\mathrm{n}} \mathrm{h}_{\mathrm{n}}$ and assuming $\mathrm{h}_{\mathrm{i}}=\mathrm{h}_{\mathrm{n}}=\mathrm{h}$, the leakage coefficients for the $\mathrm{CM}$ finite difference method become:

$$
\mathrm{C}_{\mathrm{in}}=\frac{\mathrm{A}_{\mathrm{in}}}{h \mathrm{~V}_{\mathrm{i}}} \frac{2}{\left(\frac{\mu_{\mathrm{i}}}{D_{\mathrm{g}}^{g}}+\frac{\mu_{\mathrm{n}}}{D_{n}^{g}}\right)}
$$

To calculate the average mesh flux values, we integrate equation (2.13) over the mesh volume and rearrange to get

$$
\oiint_{i}^{-g}=\frac{1}{V_{i}} \int_{V_{i}} \phi^{g}(r) d r=\phi_{i}^{g}-\frac{h^{2} \mu_{i}^{2}\left(1-\mu_{i} / 3\right)}{8 D_{i}^{g}} \sum_{n=1}^{N} C_{i n}^{g}\left(\phi_{i}^{g}-\phi_{n}^{g}\right)
$$

and the coarse mesh finite difference equation for neutron group $g$, mesh $i$ is:

$$
\sum_{n=1}^{N} C_{i n}^{g}\left(\phi_{1}^{g}-\phi_{n}^{g}\right)+R_{i}^{g} \phi_{i}^{g}-R_{i}^{g} \frac{h^{2} \mu_{i}^{2}\left(1-\mu_{i} / 3\right)}{8 D_{1}^{g}} \sum_{n=1}^{N} C_{i n}^{g}\left(\phi_{1}^{g}-\phi_{n}^{g}\right)=P_{i}^{g}
$$

or

$$
\sum_{n=1}^{N} C_{i n}^{g} f_{1}^{g}\left(\phi_{1}^{g}-\phi_{n}^{g}\right)+R_{1}^{g} \phi_{1}^{g}=P_{1}^{g}
$$

where 
User's Manual for GULDA (U)

$$
f_{i}^{g}=1-\frac{R_{i}^{g} h^{2} \mu_{i}^{2}\left(1-\mu_{i} / 3\right)}{8 D_{i}^{g}}
$$

In fact, the coarse mesh equation becomes identical to the standard equation if $f_{i}$ and $\mu_{i}$ are set to 1 and the mesh averaged flux is set equal to the mesh centered flux. The quantity $\mu_{i}$ is selected by a "rule of thumb" that guarantees $\mathrm{f}_{\mathrm{i}}>0$ for numerical stability. To determine $\mu_{i}$, consider a simpie problem of one point per hex with $P_{i}$ $=0$ :

$$
\sum_{n=1}^{6} \frac{A_{i n}}{h V_{i}} \frac{2}{\left(\frac{\mu_{i}}{D_{1}^{g}}+\frac{\mu_{n}}{D_{n}^{g}}\right)} f_{1}^{g}\left(\phi_{1}^{g}-\phi_{n}^{g}\right)+R_{i}^{g} \phi_{1}^{g}=0
$$

Solving equation (2.18) for $f_{i}$ we have:

$$
f_{1}^{g}=\frac{\mu_{i}\left(p_{1}^{g}\right)^{2}}{6 G_{1}^{g}}
$$

where

$$
\rho_{1}^{g}=h_{i} \sqrt{\frac{R_{1}^{g}}{D_{1}^{g}}} ; \quad G_{1}^{g}=\frac{1}{9} \sum_{n=1}^{6}\left(\frac{\phi_{n}^{g}}{\phi_{1}^{g}}-1\right)
$$

The value of $G_{i}$ can be obtained by considering a square, bare, homogeneous reactor containing the material in cell $\mathrm{i}$, a fixed source on two of the four sides, and zero flux on the other two sides. Analytical solution for this problem with one neutron group gives the expression for $G_{i}$ :

$$
G_{i}=\frac{1}{3} \cosh \frac{\rho_{i}}{\sqrt{2}}+\frac{2}{3} \cosh \frac{\sqrt{6}}{4} \rho_{i} \cosh \frac{\sqrt{2}}{4} \rho_{i}-1
$$

By comparing equations (2.17), (2.19), and (2.20), the equation for $\mu_{\mathrm{i}}$ 
is:

$$
\mu_{i}^{3}-3 \mu_{i}^{2}-\frac{6}{G_{i}^{g}} \mu_{i}+\frac{24}{\left(\rho_{1}^{g}\right)^{2}}=0
$$

Since $\rho_{i}$ is positive by definition and $G_{i}>0$ from equation 2.21 ( $\cosh x$ $>0$ for any value of $x$ ), the sign of $f_{i}$ depends on the sign of $\mu_{i}$ as shown in equation (2.19). Thus, if $\mu_{\mathrm{i}}$ is selected as the smallest root of equation (2.22) with $0 \leq \mu_{\mathrm{i}} \leq 1$, the physical meaning of $\mu_{\mathrm{i}}$ and the requirement of positive $f_{i}$ are both satisfied. In GILDA, equations $(2.20),(2.21),(2.22)$ are used to calculated $\rho_{i}, G_{i}, \mu_{i}$, and then equation $(2.17)$ is used to calculate $f_{i}$.

\section{Method of Solution}

The basic computational strategy employed in GILDA is to estimate the production term $\mathrm{P}$ and then solve the difference equation for each neutron group in sequence. The estimate of the production term is called the outer iteration and the estimate of the neutron fluxes is called the inner iteration. In order to speed the convergence of the production term, an over-relaxation procedure is used. The iteration strategy of GILDA is described below.

\section{III.1 Outer Iteration}

To describe the outer iteration strategy $y^{5-6}$, the system of finite difference equations is rewritten in a simple matrix form:

$$
C \phi=A \phi+B^{\prime}
$$

where $\mathrm{C}$ represents the diffusion, removal, and scattering operator, A represents the fission production operator, and B' represents the source term. If $\mathrm{j}$ is the outer iteration index, the solution to equation (3.1) is iterated as

$$
C \phi^{j+1}=A \phi^{j}+B^{\prime}=P^{j}+B^{\prime}
$$


The new production term then becomes:

$$
P^{j+1}=A \phi^{j+1}=A\left[C^{-1}\left(P^{j}+B^{\prime}\right)\right]=M P^{j}+B
$$

Equation (3.3) is an eigenvalue equation with the eigerivector $P$. The iteration matrix $M$ has a set of eigenvalues $\lambda_{k}$ and eigenvectors $\psi_{k}$ such that:

$$
\lambda_{\mathrm{k}} \psi_{\mathrm{k}}=\mathrm{M} \psi_{\mathrm{k}}
$$

The error after iteration $\mathrm{j}$ is defined by

$$
\varepsilon^{j}=p^{j}-p^{\infty}
$$

Since $\mathrm{P}^{\infty}$ is the solution of equation 3.3 , the errors satisfy the homogeneous equation.

$$
\varepsilon^{j+1}=M \varepsilon^{j}
$$

The error vector is expanded as

$$
\varepsilon^{j}=\sum_{k} \alpha_{k}^{j} \psi_{k}
$$

where coefficients $\alpha_{\mathrm{k}}^{0}$ are determined by the initial production $\mathrm{P}^{0}$. Substituting equation (3.7) into (3.6) and from (3.4) we have

$$
\begin{aligned}
& \varepsilon^{j+1}=\sum_{k} \alpha_{k}^{j+1} \psi_{k}=\sum_{k} \alpha_{k}^{j} \lambda_{k} \psi_{k}=\sum_{k} \alpha_{k}^{0}\left(\lambda_{k}\right)^{j+1} \psi_{k} \\
& \text { or } \quad \alpha_{k}^{j+1}=\lambda_{k} \alpha_{k}^{j}=\alpha_{k}^{0}\left(\lambda_{k}\right)^{j+1}
\end{aligned}
$$

Equation (3.8) shows that the outer iteration will converge only if all the absolute values of eigenvalues are less than 1; the rate of convergence is determined by the largest eigenvalue. For the neutron diffusion equation, Reference 6 shows that all eigenvalues are less than one and that convergence can be accelerated by over- 
User's Manual for GULA (U)

relaxing the production, that is:

$$
P^{j+1}=P^{j}+\omega\left[M P^{j}+B-P^{j}\right]
$$

where $\omega$ is the over-relaxation factor and $1 \leq \omega \leq 2$. From equation (3.9), equation (3.8) for the over-relaxation procedure then becomes:

$$
\alpha_{k}^{j+1}=\alpha_{k}^{j}\left[1+\omega\left(\lambda_{k}-1\right)\right]=\alpha_{k}^{0}\left[1+\omega\left(\lambda_{k}-1\right)\right]^{j+1}
$$

Comparison of equation (3.8) and (3.10) illustrates that overrelaxation corresponds to a shift in the eigenvalues from $\lambda_{k}$ to $\left[1+\omega\left(\lambda_{k}-1\right)\right]$. The eigenvalues are ordered such that

$$
1 \geq \lambda_{1} \geq \lambda_{2} \geq \ldots \ldots \ldots \geq \lambda_{n} \geq \ldots \ldots \ldots \geq \lambda_{N} \geq 0
$$

As the number of iterations increase, $\mathrm{j}$ becomes large, the error can be approximated as:

$$
\varepsilon^{j}=\alpha_{1}^{0}\left[1+\omega\left(\lambda_{1}-1\right)\right]^{j} \psi_{1}
$$

and the error on the next iteration could be zero if:

$$
1+\omega\left(\lambda_{1}-1\right)=0 \Rightarrow \omega=\frac{1}{1-\lambda_{1}}
$$

Only one iteration can be done with this value since repeated iterations would amplify the node near $\lambda_{N}$ and the solution would rapidly diverge as shown in equation (3.10). When the number of iterations is not sufficiently large such that equation (3.11) is valid, convergence can be accelerated by selecting an optimum value of $\omega$ which will shift the effective eigenvalues such that

$$
\left[1+\omega\left(\lambda_{1}-1\right)\right]=-\left[1+\omega\left(\lambda_{N}-1\right)\right]
$$

and from equation (3.13) with $\lambda_{N}=0$ we have: 


$$
\omega=\frac{2}{2-\lambda_{1}}
$$

The value of $\lambda_{1}$ need not be computed directly since its value can be inferred by some suitable norm of the error vector from equation (3.11), e.g.:

$$
\begin{aligned}
& \frac{\left\|\varepsilon^{j+1}\right\|}{\left\|\varepsilon^{j}\right\|}=\frac{\left\|\delta^{j+1}\right\|}{\left\|\delta^{j}\right\|}=\Delta^{j} \rightarrow\left[1+\omega\left(\lambda_{1}-1\right)\right] \\
& \lambda_{1}=\frac{\Delta^{j}-1}{\omega}+1
\end{aligned}
$$

where $\delta^{j}=\mathrm{P}^{\mathrm{j}}-\mathrm{P}^{\mathrm{j}-1}$

In GILDA, $\omega=1$ is used for the first three iterations. After that, $\Delta^{j}$ of the five most recent iterations are calculated and the procedure is processed as follows:

If largest $\Delta$ - smallest $\Delta$ is larger than 0.05 , the old value of $\nu$ is used for the next iteration.

If $0.01<$ largest $\Delta$ - smallest $\Delta \leq 0.05$ then equation (3.14) is used to calculate $\omega$ for the next iteration.

If largest $\Delta$ - smallest $\Delta \leq 0.01$ and the last $\omega$ was calculated from equation (3.12), then equation (3.14) is used to calculate $\omega$ for the next iteration.

If largest $\Delta$ - smallest $\Delta \leq 0.01$ and the last $\omega$ was not calculated from equation (3.12), then equation (3.12) is used to calculate $\omega$ for the next iteration.

\section{III.3 Inner Iteration}

To calculate point flux values at each outer iteration step, the Liebmann method ${ }^{3}$ of inner iteration is used. During the inner iteration, each energy group of neutron flux is computed one at a time, starting with the highest energy group $(\mathrm{g}=1)$. For each group, two mesh sweeps are made using equation (2.16) for coarse mesh finite difference or equation (2.11) for standard finite difference 
method. Suppose for a standard finite difference method, from equation (2.11) we have a solution of neutron fluy for each mesh point:

$$
\phi_{1}^{g, j+1}=\frac{P_{1}^{g, j}+\sum_{n 1} C_{n 1}^{g} \phi_{n}^{g, j+1}+\sum_{n 2} C_{n 2}^{g} \phi_{n}^{g, j}}{R_{1}^{g}+\sum_{n} C_{n}^{g}}
$$

where $\mathrm{j}$ is an iteration index, $\mathrm{i}$ is a mesh point index, $\mathrm{nl}$ refers to neighbor points of $\mathrm{i}$ which have been computed during this sweep, $\mathrm{n} 2$ refers to neighbor points of $\mathrm{i}$ which have not yet been computed during this sweep, and $n$ refers to all neighbor points of $i$. The first sweep traverses the mesh points spiraling radially outward from the center in a clockwise direction and the second sweep reverses this direction. Once the new flux values are calculated, the production term will be updated by these new flux values and convergence checks are performed. The iteration stops if $\mathbf{k}$ effective and production convergence criterions are both satisfied; otherwise, the next step of outer iteration begins. Figure 1 in the next page shows the flow chart of overall iteration strategy in module GILDA. 


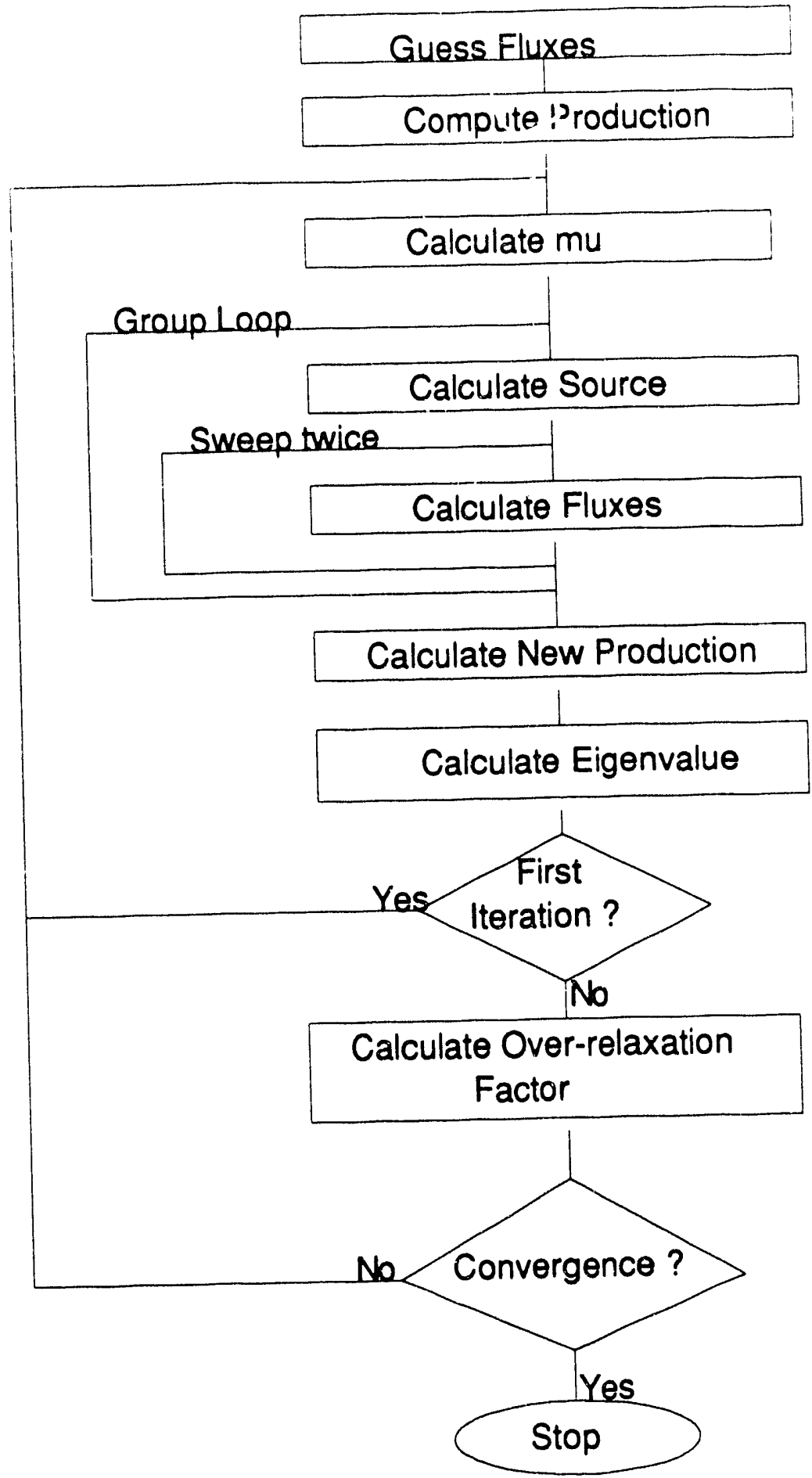

Figure 1: Over-all iteration strategy 


\section{Program Subroutines}

GILDA is composed of seven subroutines: MAIN, NEIGH, INPUT, WORK, RMUNU, RELAX2, and EDIT. The functions of these subroutines are described below:

1. The MAIN routine calls subroutine NEIGH to produce the special geometry records for problems of forty nine hex lattice. It then reads record INPUT.GILDA.JOB.jsiqwr(), which defines the number and names of cases to be executed, and two optional records, INPUT.GILDA.EDIT.jsiqwr() and INPUT.GILDA.CONVERG.jsiqwr() for the editing option and convergence criterion's. For each specified case, MAIN calls INPUT to process the input data and calls WORK to perform the calculations sequentially.

2. Subroutine NEIGH produces special geometry records SYSTEM.BIGGILDA.TRANS. number (where number is the number of points per hex) for "big" lattice (forty nine hexes) problems.

3. Subroutine INPUT reads the INPUT.GILDA.CASE.casename record for general information, material names, and few group cross section record names of those materials. The routine then reads FEWGRP.namel name2.name3.MACRO.name4 records for the fewgroup cross sections and assigns these cross sections to the corresponding hexes in the lattice. Finally, the routine reads record SYSTEM.GILDA.TRANSROT.number or SYSTEM.GILDA.TRANS.number or SYSTEM.BIGGILDA.TRANS.number for the lattice geometry descriptions.

4. Subroutine WORK performs the iterative calculations for eigenvalues (effective multiplication factor or buckling) and the point flux values. WORK calls RMUNU to calculate $\mu$ and calls REIAX2 to calculate over-relaxation factors during the iteratiun. After solutions of each search converge, WORK calls EDIT to process and print the results. As many as five searches may be specified in a single case. 
5. Subroutine RMUNU calculates $\mu$ from equation (2.22).

6. Subroutine RELAX2 calculates over-relaxation factors from equations (3.14) and (3.12).

7. Subroutine EDIT writes the point flux values to BIGGILDA.PFLUXES.casename.group.jsiqwr() records, calculates the total fluxes in a hex and writes the results to GILDA.CELLFLUX.casename.jsiqwr() records. The routine also caiculates the normalized cell fluxes, normalized point fluxes, the average supercell group fluxes, the material fluxes, the supercell averaged cross sections, and writes these to the defined output file.

\section{Input Description}

This section provides general instructions on setting up the different types of problems for GILDA by describing each JOSHUA input record. Users who are not familiar with the JOSHUA system should review the JOSHUA manual ${ }^{3}$ for the conventions and terminology used. The sample input records are shown in Appendix $C$, and instructions to run GILDA on the VAX and IBM are shown in Appendix B.

INPUT.GILDA.JOB.jsiqwr() : this record is created by the user, one record is required per job and is located in the user data set.

Data: NCASEX,(DCASE(NCASE),NCASE=1,NCASEX)

NCASEX: Number of cases run for this job (20 maximum) DCASE: Case names by case.

INPUT.GILDA.EDIT.jsiqwr() : This record is optional. It is created by the user if desired.

Data: NEDIT

NEDIT [0]: Output option: (0-Print out all input data, 2-print out few group cross section only). 
INPUT.GILDA.CONVERG.jsiqwr()： This record is optional. It is created by the user if desired.

\section{Data: CONNF,CONNK,CONVB,ICONVB}

CONNF [5.E-05]: Production convergence criterion CONNK [5.E-05]: $\mathrm{k}$ effective convergence criterion CONVB [5.E-08]: Buckling convergence criterion ICONVB [0]: Buckling convergence type (0-Absolute, 1Relative).

INPUT.GILDA.CASE.casename: These records are created by the user, one record is required per case. The records must be located in the user data set.

Data: NCLX,IAPROX,NGIX,NHPX,NSRCX,PITCH,(TYPE(I),DBSQD(I), $\operatorname{DXKEFF}(\mathrm{I}), \mathrm{I}=1,5)$,WTRROT,WTR,(FG(1,J),J=1,4),WCL(7), $\mathrm{FG}(2, \mathrm{~J}), \mathrm{J}=$ 1,4),WCL(6),WCL(2), $\mathrm{FG}(3, \mathrm{~J}), \mathrm{J}=1,4), \mathrm{WCL}(1),(\mathrm{FG}(4, \mathrm{~J}), \mathrm{J}=1,4), \mathrm{WCL}(5)$ ,WCL(3),(FG(5,J),J=1,4),WCL(4),(FG(6,J),J=1,4),(FG(7,J),J $=1,4)$

NCLX: Number of hexes ( 7 or 49 ) IAPROX: Approximation method (0-STD, 2-CM)

NGIX: Number of energy groups ( 1 to 10 )

NHPX: Number of points per hex $(1,3$, or 6$)$

NSRCX: Number of searches (1 to 5)

PITCH: Hexagonal pitch (inch)

TYPE: Search type by search (K-k effective, B-buckling) DBSQD: Buckling by search

DXKEFF: K-effective by search

WTRROT: Translation and rotational symmetry (blank-no, filled-yes)

WTR: Translational symmetry only (blank-no, filled-yes)

FG: Few group cross section record names by cell

WCL: Material names by cell

FEWGRP. $f g(n, 1) \cdot f g(n, 2), f g(n, 3)$. MACRO. $f g(n, 4):$ These records are created by the user, typically by running GLASS. One record is required for each unique cell type in the lattice. These records must be located in the user data set. 
Data: NG,(XD(I),XC(I),XF(I),XP(I),I=1,NG),((XS(I,J),I=1,NG),J=1,NG), $((\mathrm{XCHI}(\mathrm{I}, \mathrm{J}), \mathrm{I}=1, \mathrm{NG}), \mathrm{J}=1, \mathrm{NG})$

NG: Number of energy groups (maximum 10)

XD: Capture cross sections by group

XF Fission cross sections by group

XP: Production cross sections group

XS: Scattering cross section matrix

$\mathrm{XCHI}$ : Fission spectrum matrix (all fission neutrons are in fast group).

SYSTEM.GILDA.TRANSROT.nhpx : These records are stored in the standard data set. The user need not create these records.

Data: NBRQ,NPTQ,(('RRPT(NBR,NPT),NBR=1,NBRQ),NPT=1,NPTQ)

NBRQ: Number of neighbors per point $(6,4$, or 3$)$

NBTQ: Number of total mesh points $(7,21,42,49,147$, or 294)

NBRPT: Index of up to six neighbor mesh points by mesh point.

SYSTEM.GILDA.TRANS.nhpx : These records are stored in the standard data set. The user need not create these records.

Data: NBRQ,NPTQ,((NBRPT(NBR,NPT),NBR=1,NBRQ),NPT=1,NPTQ)

NBRQ: Number of neighbors per point $(6,4$, or 3$)$

NBTQ: Number of total mesh points $(7,21,42,49,147$, or 294)

NBRPT: Index of up to six neighbor mesh points by mesh point.

SYSTEM.BIGGILDA.TRANS. $n h p x$ : These records are stored in the user data set by subroutine NEIGH. The user need not create these records

Data: NBRQ,NPTQ,((NBRPT(NBR,NPT),NBR=1,NBRQ),NPT=1,NPTQ)

NBRQ: Number of neighbors per point $(6,4$, or 3$)$

NBTQ: Number of total mesh points $(7,21,42,49,147$, or 294)

NBRPT: Index of up to six neighbor mesh points by mesh point. 
User's Manual for GllDA (U)

\section{Output Description}

This section describes JOSHUA records output, meanings of the output results, and the error messages that may be encountered during execution.

\section{VI.1 JOSHUA Output Records}

BIGGILDA.PFLUXES.casename.ngf.jsiqwr(): There is one record for each neutron group of each case with $n g f$ being the neutron group number. These records contain the calculated point flux values of the entire lattice.

Format: NGF,NPTX ,(FLUX(NGF,NPT),NPT=1,NPTX )

NGF: Neutron energy group number

NPTX: Total number of points in the lattice

FLUX: Point flux values by group, by point.

GILDA.CELLFLUX.casename.jsiqwr()：There is one record for each case. These records contain k-effective, buckling, and calculated hex flux values of hexes in the lattice.

Format: NCLX,NGLX,XKEFF,BSQD,(NCL,(CFLUX(NGF,NCL),NGF=1,NGIX), $\mathrm{NCL}=1, \mathrm{NCLX})$

NCLX: Number of hexes

NGIX: Number of neutron energy groups

XKEFF: Effective multiplication factor

BSDQ: Buckling

CFLUX: Hex flux values by group, by hex

The hex flux values are calculated as below:

$$
\Phi_{h}^{g}=\sum_{i=1}^{I} \phi_{1, h}^{g}
$$

where $h$ is the hex number, $i$ is point number, and $I$ is number of 
points (meshes) in hex $h$. Note that the hex flux values here are not the avsrage flux of a hex, but the hex average flux times number of points per hex.

\section{VI.2 Additional Outputs}

Besides the two JOSHUA output records above, if the output option NEDIT is set to 0 , the program also calculates normalized cell fluxes, normalized point fluxes, average supercell group flüies, material fluxes, supercell averaged cross sections, and prints these to the defined output file. The normalized "cellwise" flux values for a forty nine hex lattice are:

$$
\bar{\Phi}_{c}=\frac{7 \sum_{h=1}^{7} \sum_{i=1}^{1} \phi_{i, h, c}^{g}}{\sum_{c=1}^{7} \sum_{h=1}^{7} \sum_{i=1}^{1} \phi_{i, h, c}^{G}}
$$

where $c$ is cell number ( 1 to 7 ), $h$ is hex number in cell $c$ ( 7 hexes per cell), $i$ is point number in hex $h(1,3$, or 6 points per hex), and $G$ is the last energy group (lowest) number. For a seven hex lattice, a cell is also a hex and equation (5.2) for normalized cellwise flux values becomes:

$$
\bar{\Phi}_{\mathrm{c}}=\boldsymbol{\Phi}_{\mathrm{h}}=\frac{7 \sum_{\mathrm{i}=1}^{\mathrm{g}} \phi_{\mathrm{i}, \mathrm{h}}^{\mathrm{g}}}{\sum_{\mathrm{h}=1}^{7} \sum_{\mathrm{i}=1}^{\mathrm{I}} \phi_{\mathrm{i}, \mathrm{h}}^{\mathrm{G}}}
$$

The normalized point flux values are: 


$$
\stackrel{-g}{\phi_{i}}=\frac{7 \phi_{i}^{g}}{\sum_{i=1}^{N P} \phi_{i}^{G}}
$$

where NP is total number of points (meshes) in the lattice. The average supercell group fluxes are:

$$
\overline{\boldsymbol{\Phi}}=\frac{\sum_{i=1}^{N P} \phi_{i}^{g}}{\sum_{i=1}^{N P} \phi_{i}^{G}}
$$

The material fluxes are:

$$
\boldsymbol{\Phi}_{\mathrm{m}}=\frac{\sum_{\mathrm{c}=1}^{\mathrm{C}} \boldsymbol{\Phi}_{\mathrm{c}}^{-\mathrm{g}}}{\mathrm{C}}
$$

where $m$ is type of material and $C$ is number of cells which have material $\mathrm{m}$. The supercell average cross sections are:

$$
\Sigma_{x}^{-g}=\frac{\sum_{m=1}^{M}\left[\Sigma_{x, m}^{g}\left(\sum_{c=1}^{C} \Phi_{c}^{-g}\right)\right]}{\sum_{m=1}^{M}\left[\sum_{c=1}^{C}-g / \Phi_{c}\right.}
$$




$$
\mathrm{D}=\left[\frac{\sum_{m=1}^{M}\left[\frac{1}{D_{m}^{g}}\left(\sum_{c=1}^{C} \Phi_{c}^{-g}\right)\right]}{\sum_{m=1}^{M}\left[\sum_{c=1}^{C} \Phi_{c}^{-g}\right]}\right]^{-1}
$$

where $M$ is total material types in the lattice, $\bar{\Sigma}_{x}$ is supercell average cross section of type $x(x=a$ for absorption, $c$ for capture, $f$ for fission, $r$ for removal), and $D$ is supercell average diffusion coefficient. Nc:s that group g removal cross section output here means scattering out cross section from group $\mathrm{g}$ to $\mathrm{g}+1$.

\section{VI.3 Error and Vyarning Messages}

During the execution, error stop or warning messages could be printed out. These messages are explained as below:

1. CANNOT READ INPUT.GILDA.JOB:

The required input record INPUT.GILDA.JOB.jsiqwr() was missing or illegal data were detected in this record. This record should be located in the user data set.

2. CANNOT READ- INPUT.GILDA.CASE.casename :

The required input record INPUT.GILDA.CASE.casename was missing or illegal data were detected in this record. This record should be located in the user data set.

3. ILLEGAL NO. OF ENERGY GROUPS IN RECORD INPUT.GIIDA.CASE.casename :

The record INPUT.GILDA.CASE.casename does not have a valid number of energy groups (number of energy groups should be from 1 to 10 ).

4. ILLEGAL NO. OF POINTS PER HEX IN RECORD INPUT.GILDA.CASE.casename : 
User's Manual for GULD (U)

The record INPUT.GILDA.CASE.casename does not have correct number of points per hex (number of point per hexes should be 1,3 , or 6 only).

5. ILLEGAL RECORD FOR CASE casename

FEWGRP.name1.name2.name3.MA.CRO.name4 :

The few group cross section recorc FEWGRP.name1.name2. name 3.MACRO.name4 for case casename was missing or illegal data were detected in this record. This record is located in the user data set.

6. INCONSISTENT GROUP STRUCTURE IN RECORDS INPUT.GILDA.CASE.casename FEWGRP.name 1.name2.nam:3.MACRO.name4 :

The number of energy groups in INPUT.GILDA.CASE.casename and FEWGRP.name1.name2 .name3.MACRO.name4 records of case casename are not identical.

7. CASE casename HAS ONE OR MORE CELLS WITH ILLEGAL MATERIAL ASSIGNMENT:

One or more cells have incorrect material name. check INPUT. GILDA.CASE.casename record for the material types.

8. ILLEGAL SEARCH TYPE FOR SEARCH number,CASE casename :

Search type of search number is not correct. Only two types of search are permitted in GILDA: $K$ for $k$ effective and $B$ for buckling. Check INPUT.GILDA.CASE.casename record for this search numter.

9. CONVERGENCE FAILED

Solution is not converged after maximum number of iterations (100). 
User's Manual for GuLDA (U)

\section{Appendix A: Tables and Figures}

\section{A. 1 Table}

\section{GEOMETRY COEFFICIENTS}

\begin{tabular}{|c|c|c|c|c|}
\hline $\begin{array}{l}\text { Point } \\
\text { per hex }\end{array}$ & Mesh pitch & Mesh & & Mesh \\
\hline $1 P$ & & $\mathrm{P} / \sqrt{3}$ & $\sqrt{3}$ & $\mathrm{P}^{2} / 2$ \\
\hline $3 P / 2$ & & $P / \sqrt{3}$ & $\sqrt{3}$ & $P^{2} / 6$ \\
\hline $6 \mathrm{P} / 3$ & & $P / \sqrt{3}$ & $\sqrt{3}$ & $P^{2} / 12$ \\
\hline
\end{tabular}

where $P$ is hexagonal pitch

\section{A.2 Figures}

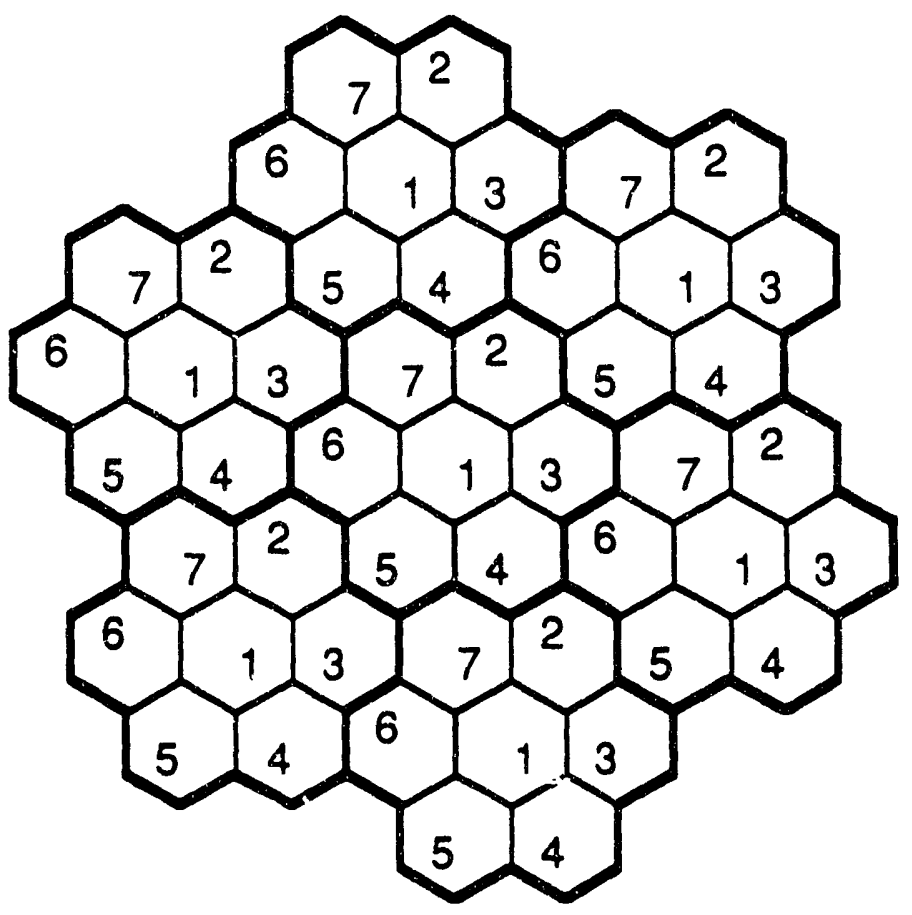

Figure 2: One point per hex. Symmetry by translation. 


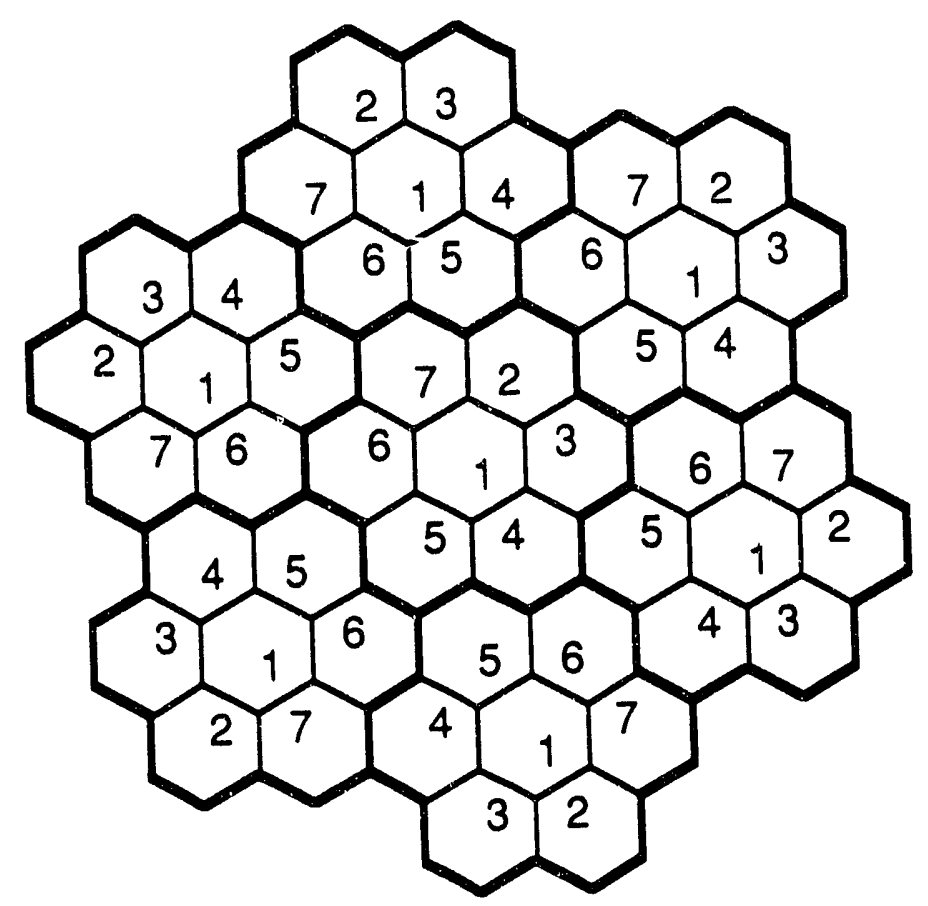

Figure 3: One point per hex. Symmetry by translation and rotation.

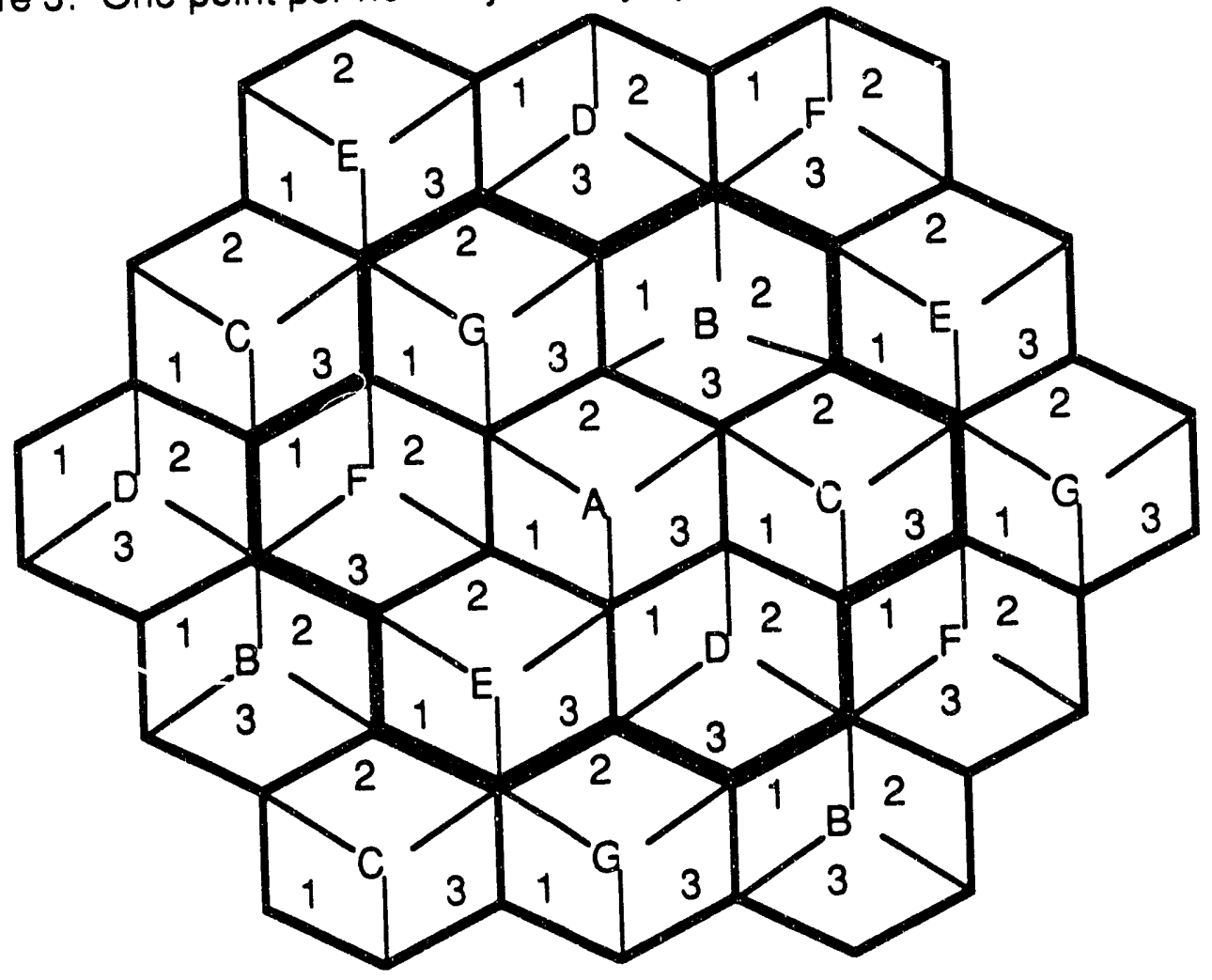

Figure 4: Three points per hex. Symmetry by translation. 


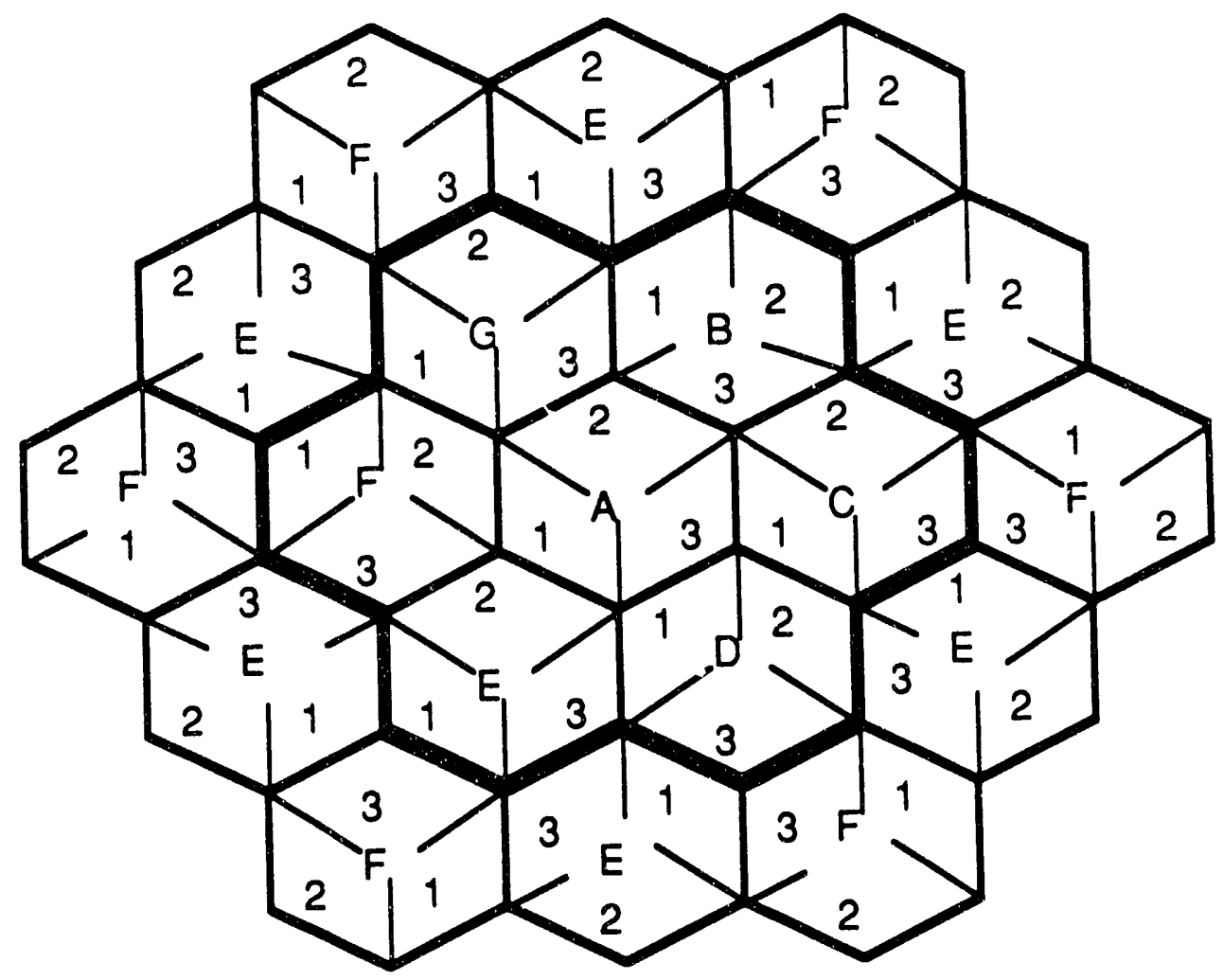

Figure 5: Three points per hex. Symmetry by translation and rotation.

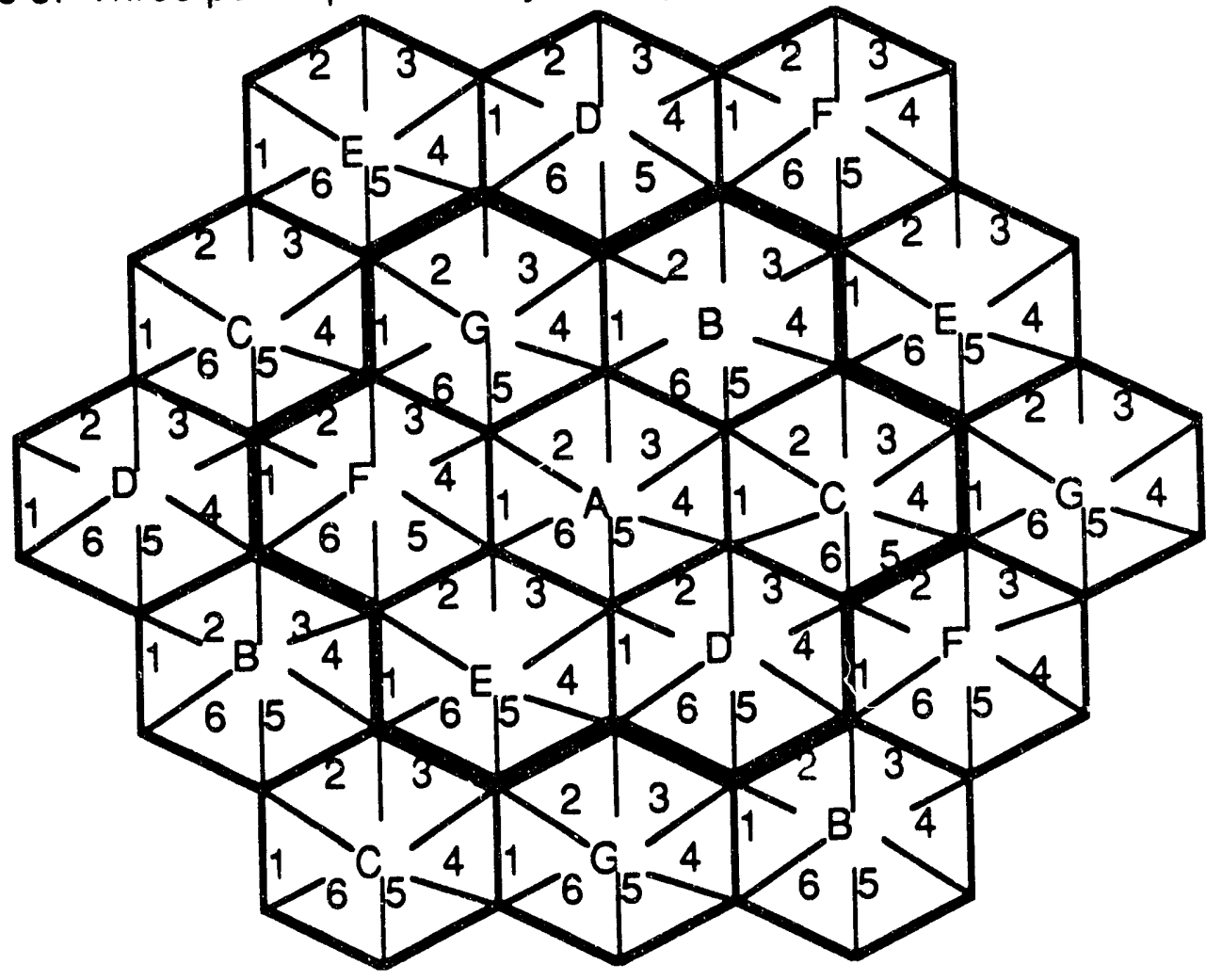

Figure 6: Six points per hex. Symmetry by translation. 


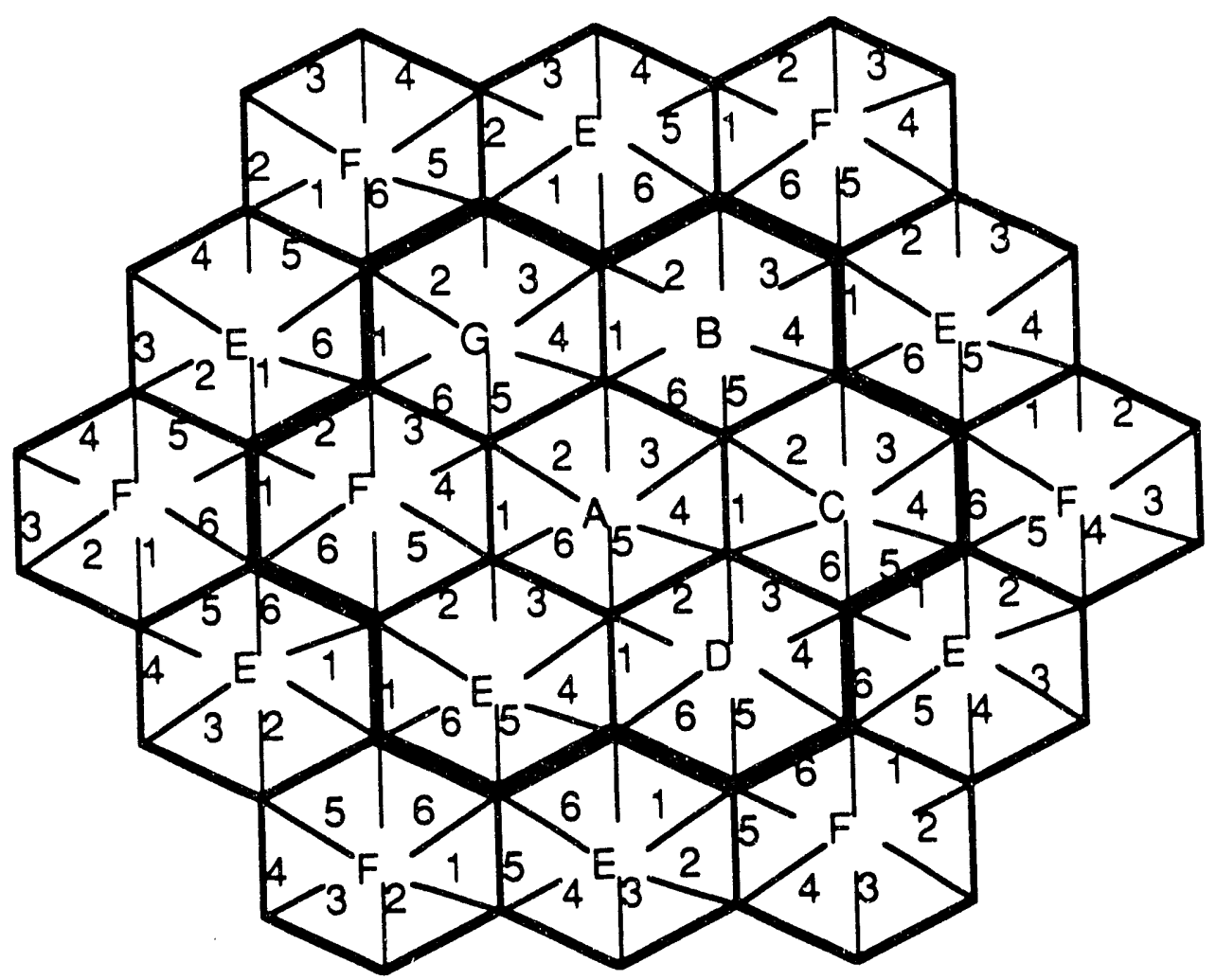

Figure 7: Six points per hex. Symmetry by translation and rotation.

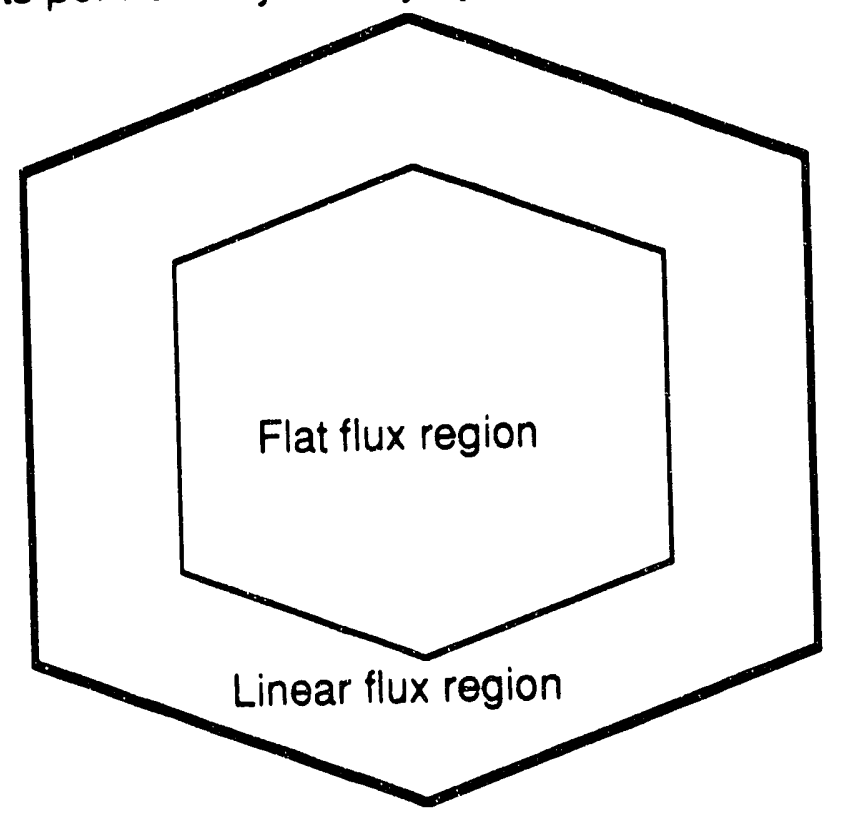

Figure 8: Geometry of Coarse Mesh for one point per hex. 


\section{Appendix B: Running GILDA on VAX/VMS and IBM Systems}

\section{B.1 Running GILDA on VAX/VMS}

GILDA is a JOSHUA module and is run under the control of the JOSHUA system. All JOSHUA jobs must begin with the execution of the JOSHUA monitor program which establishes the execution environment and then executes the desired modules. There are two methods to tell JSMON which module we want to execute: By specifying GILDA as a command parameter on the JSMON command line or by including a keyword in a JSIN file which contains instructions to the JSMON program. For the first rivethod, the JSMON command line format is:

$\$$ JSMON [/qual1/qual2/.../qualn] [module_name]

where the optional qualifiers are described in reference 2 and the module_name is GILDA.

For the second method, the JSIN command file which contains one or more qualifiers is created by the user. The JSIN file is then executed by the VMS command:

\section{$\$ \mathrm{JSMON} / \mathrm{JSIN}=$ name. $\mathrm{JSIN}$}

where name is the name of the JSIN file.

A sample JSIN file for a benchmark problem (called GIL17062.JSIN) is shown below:

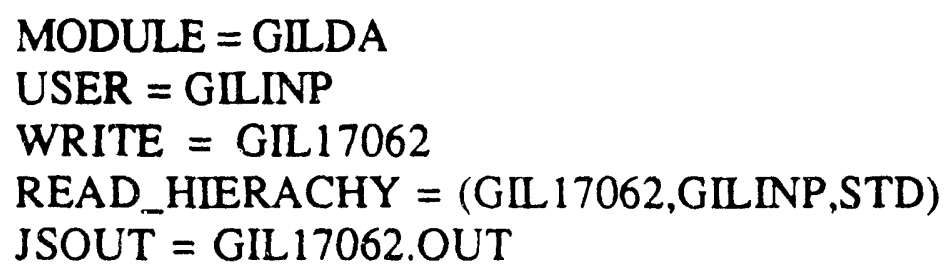

The first line of the file specifies that GILDA is the module to be run, the second line specifies the user data set name (GILBENCH), the third line specifies the name of the output (WRITE) data set that 
GILDA will create, the fourth line specifies the order in which the data sets should be searched for various records, and the last line specifies the name of the text output file generated by GILDA. These lines can be in any order.

For more details about executing a JOSHUA module, users can consult chapter 7 of reference 2 .

\section{B.2 Running GILDA on IBM}

There are two versions of JOSHUA on the IBM; the J70 (old JOSHUA) version is being phased out and the J80 (new JOSHUA) version is becoming the standard JOSHUA in use. On the IBM, jobs are submitted by using Job Control Language (JCL). Below are samples of minimum JCL for a benchmark problem with J70 and J80 JOSHUA versions. Explanation of these lines is not included here, users should consult reference 7 for the meanings of these lines.

Sample JCL for GILDA (J70)

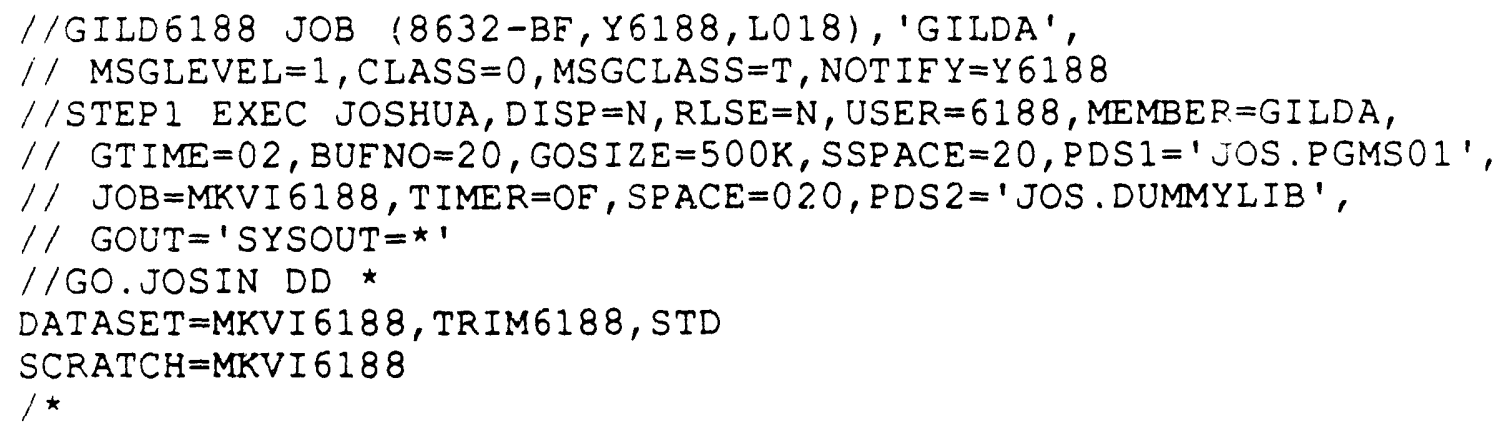




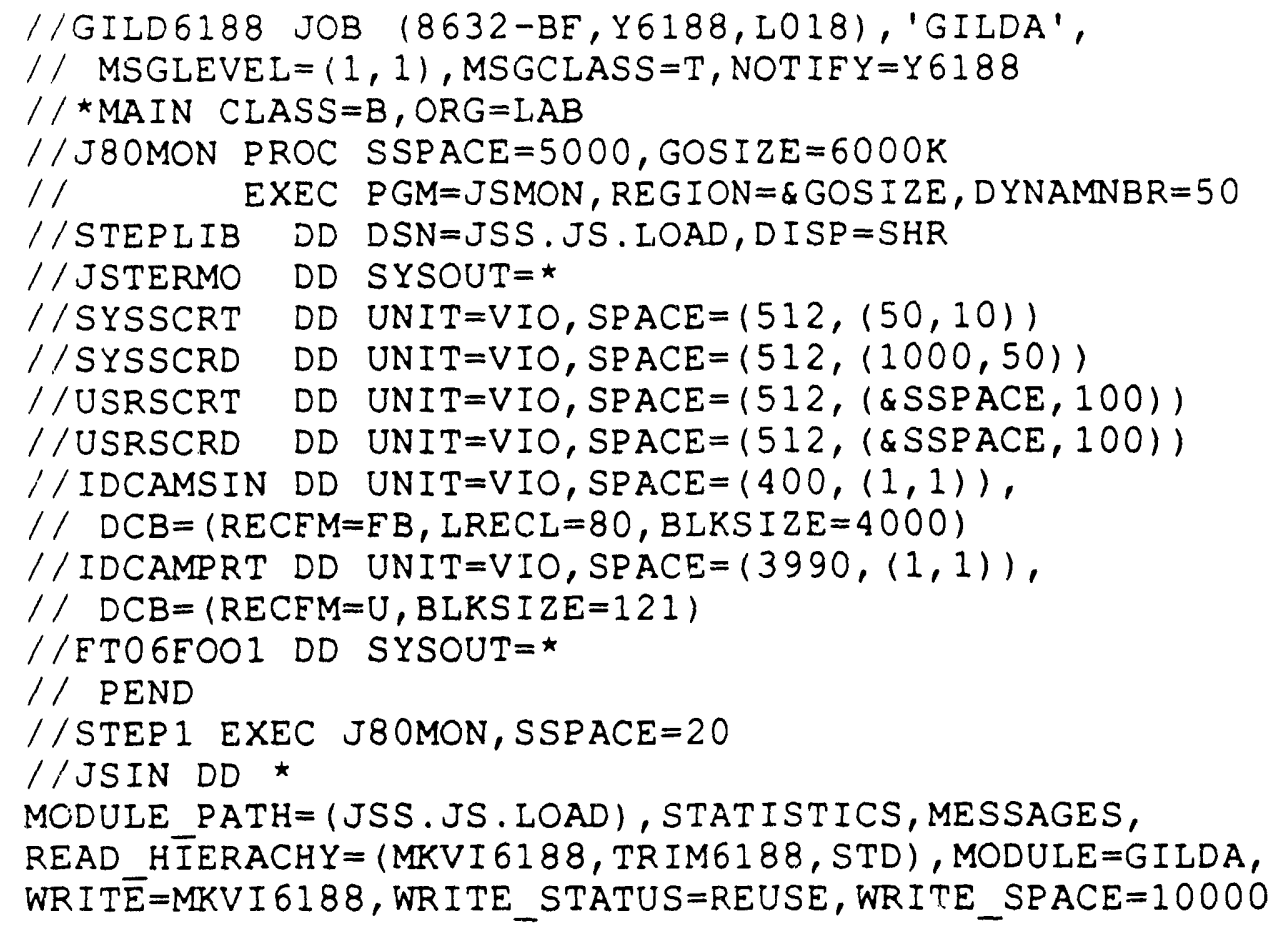


User's Manual for GILDA (U)

\section{Appendix C: Sample Input Records}

INPUT.GILDA.JOB.GIL17062

NUMBER OE GILDA CASES $=: 1$

${ }^{\mathrm{NO}} \overline{1} \frac{\mathrm{NAME}}{\mathrm{BASE}} \mathrm{NO}^{\mathrm{NO}} \mathrm{NAME} \_\mathrm{NO}_{-}{ }^{\mathrm{NAME}}$

INPUT.GILDA.CONVERG.GIL17062
PRODUCTION CONVERGENCE CRITERION $=: 50.0000 E-07$
K-EFFECTIVE CONVERGENCE CRITERION $=: 50.0000 E-07$
BUCKLING CONVERGENCE CRITERION $=: 50.0000 E-09$
BUCKLING CONVERGENCE TYPE $\quad=: 0$ (O-ABSOLUTE, 1-RELATIVE)

INPUT.GILDA.CASE.BASE

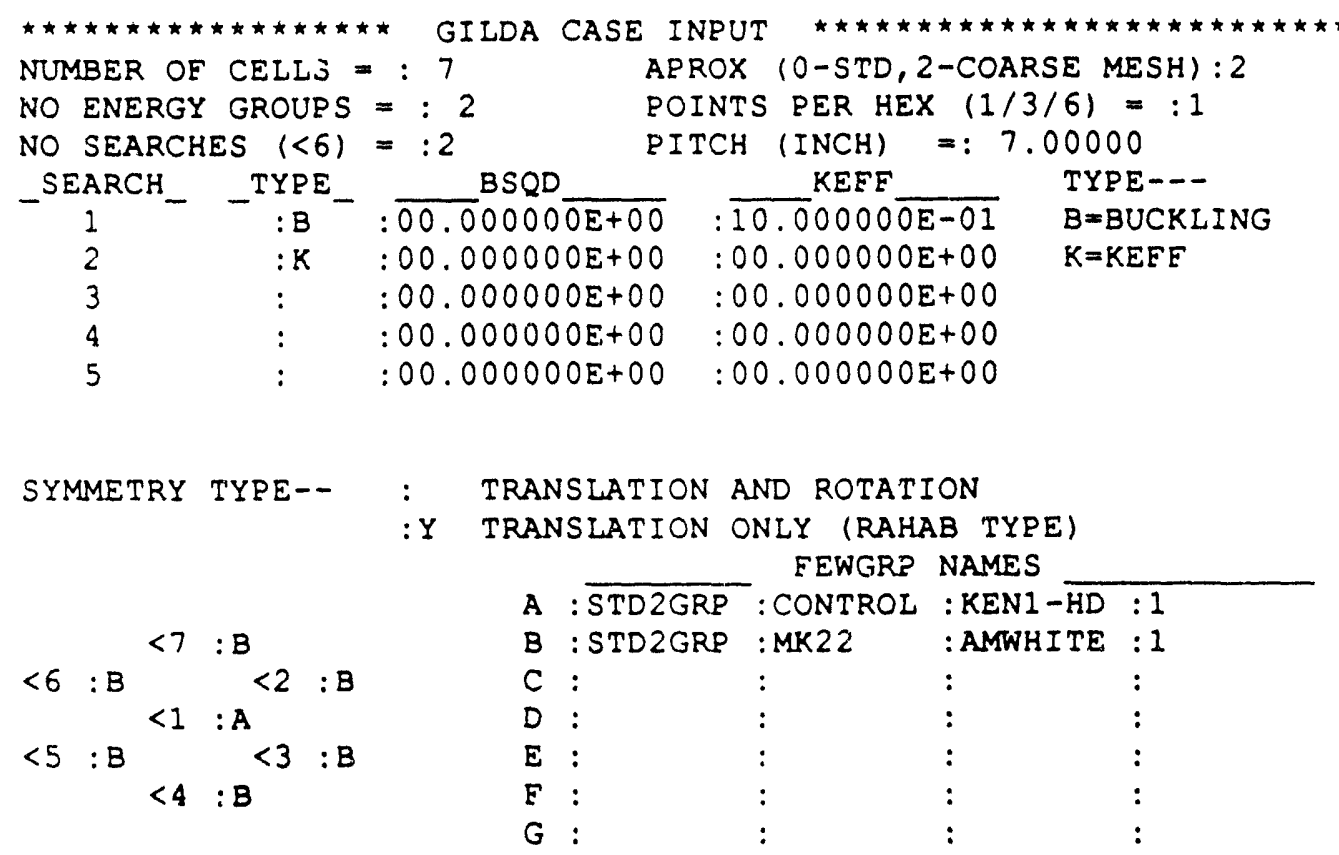

\section{FEWGRP.STD2GRP.CONTROL.KEN1-HD.MACRO.1}

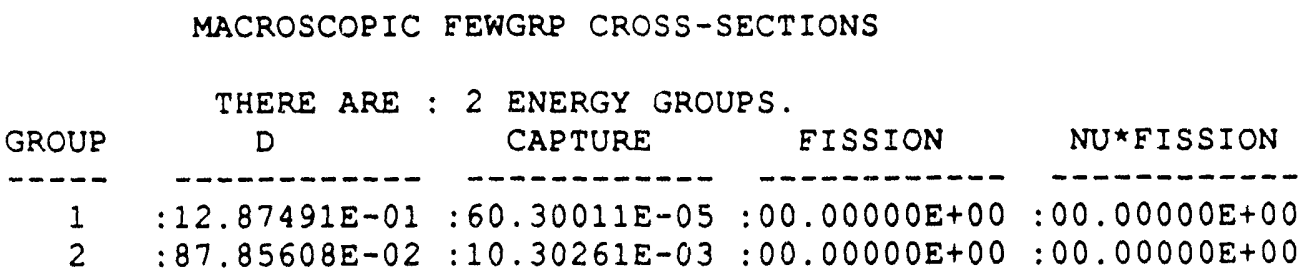




\section{User's Manual for GILDA (U)}

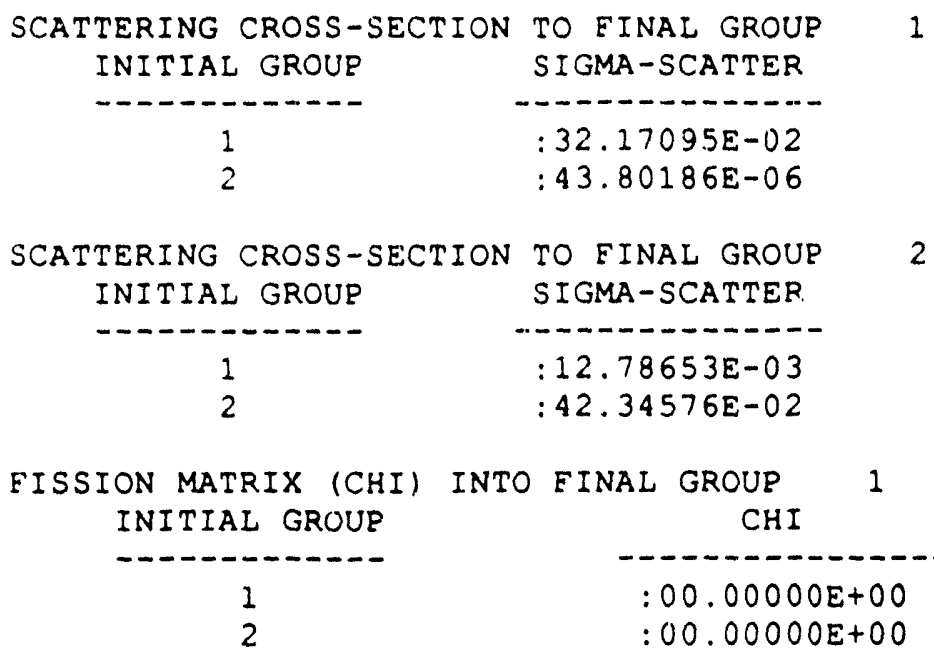

FISSION MATRIX (CHI) INTO FINAL GROUP 1 INITIAL GROUP

INITIAL GROUP 1

CHI

$: 00.00000 E+00$ $: 00.00000 E+00$

EISSION MATRIX (CHI) INTO EINAL GROUP 2 INITIAL GROUP

-

1

$\mathrm{CHI}$

2

$: 00.00000 E+00$

$: 00.00000 E+00$

FEWGRP.STD2GRP.MK22.AMWHITE.MACRO.1

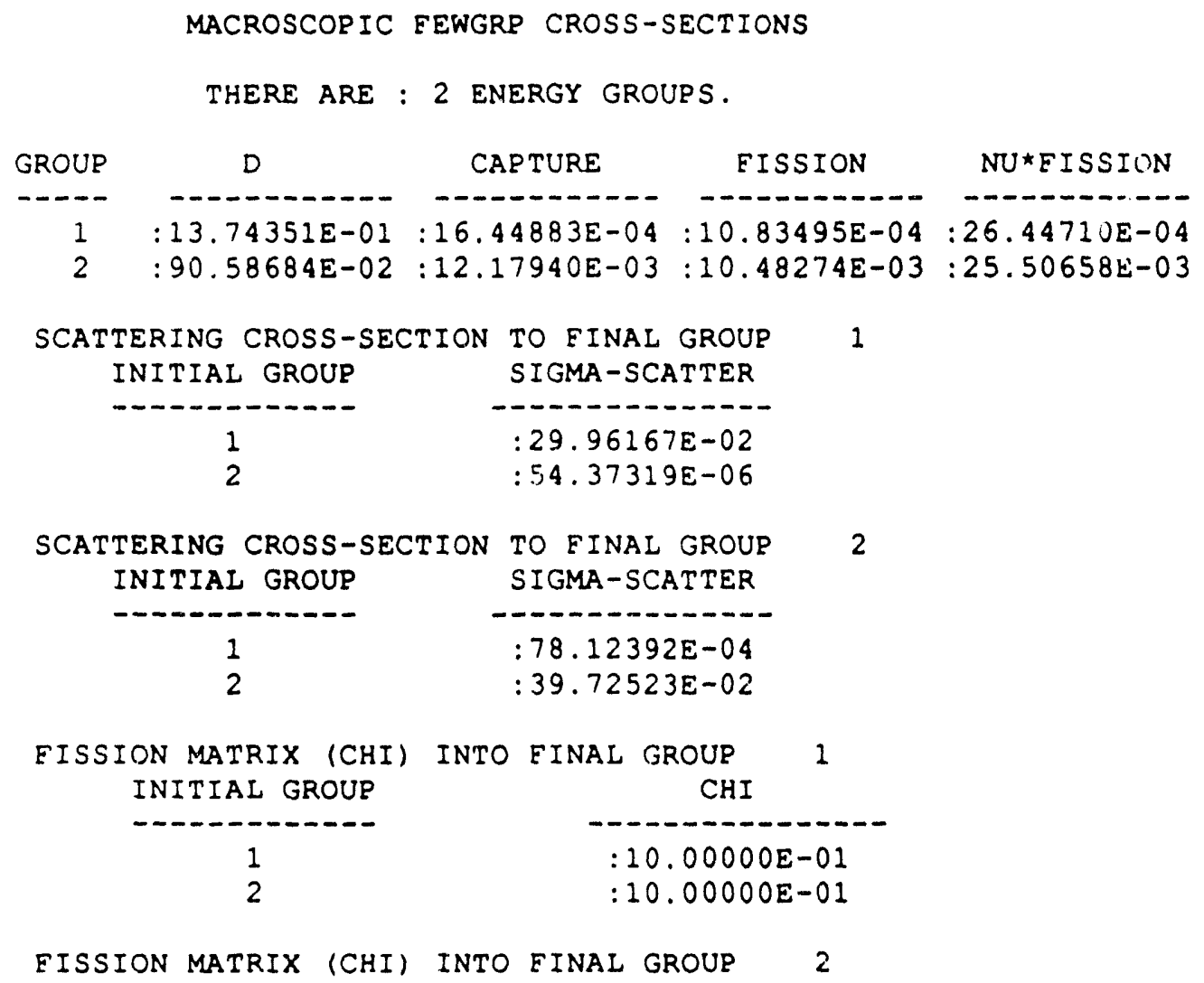


METsC

Toetionolo

On Thom

W SR C.TR.91.481

Ir on Tarom

August 1991

Page 35 of 36

User's Manual for GllDd (U)

INITIAL GROUP

INITIAL GROUP

1

GHI

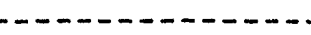

$: 00.00000 E+00$

$: 00.00000 E+00$ 


\section{References}

1. M. B. Stroud, GILDA, a memo to distribution, October 25, 1973.

2. W. H. Reed, H. C. Honeck, J. T. McCort, B. W. Westmoreland, The Joshua Users' Manual, DPSPM-GEN-36, September 14, 1987.

3. G. I. Bell, S. Glasstone, Nuclear Reactor Theory, Robert E. Krieger Publishing Co., Inc., 1970.

4. H. L. Dodds, Jr., H. C. Honeck, and D. E. Hostetler, A Coarse-Mesh Method for Multidimensional. Mixed-Lattice Diffusion Calculations, Nuclear Science \& Engineering, 62, 1977.

5. DPSTM-500 Loshua Manual, Volume 10, "Module GRIMHX Generalized Reactor Iterative Module in Hexagonal Geometry", November 1973 (Draft).

6. A. F. Henry, H. C. Honeck, The Physics of Water Reactors, Notes for a series of lectures given to the staff of the SRS, July 17-28, 1989.

7. MVS/Extended Architecture JCL User's Guide, GC28-1351-4, September 1989. 

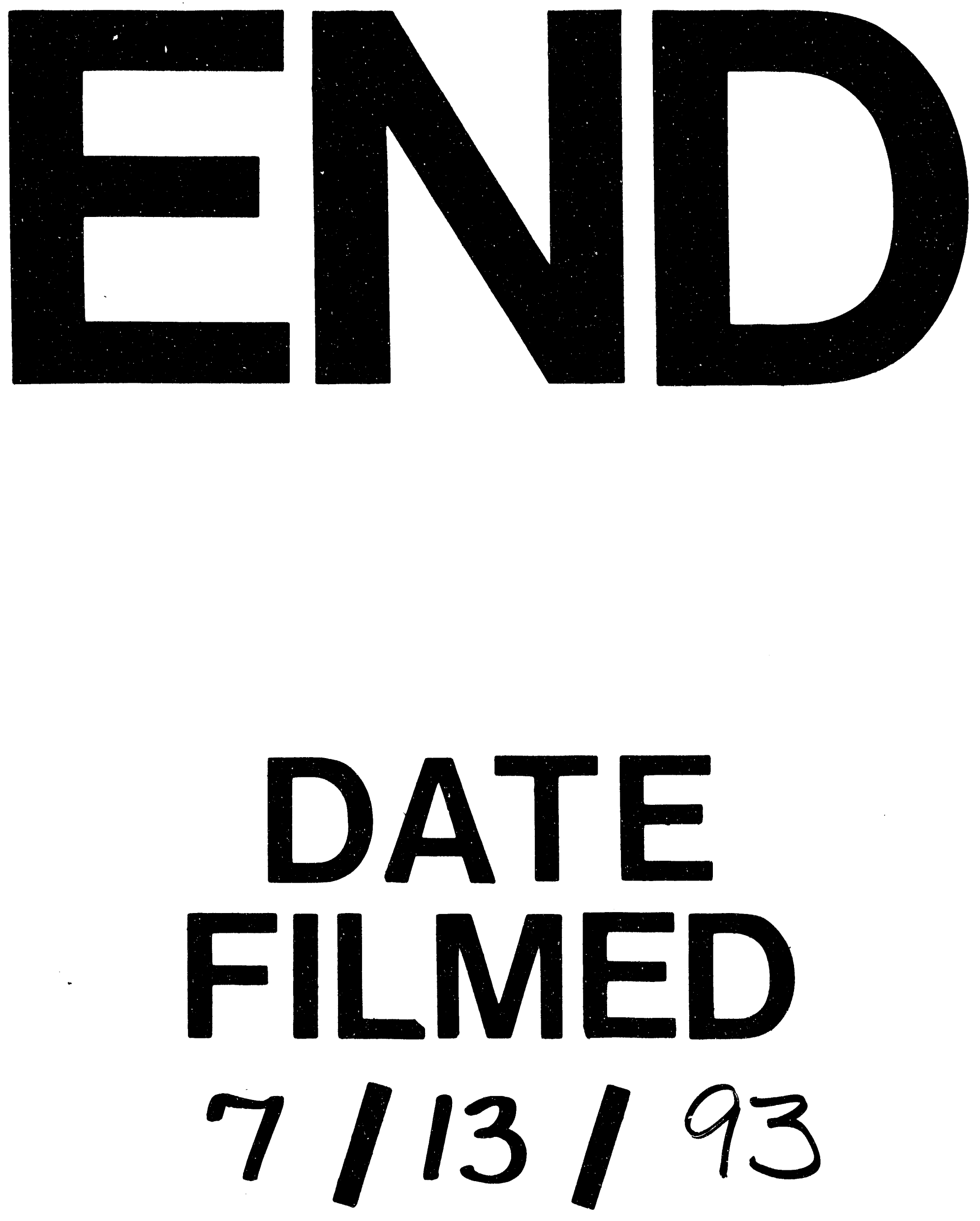
